\title{
Do interacting ultraviolet fixed point exist, and if so, what can we do with them?
}

\section{Daniel Litim*}

Department of Physics and Astronomy, University of Sussex

E-mail: d.litimesussex.ac.uk

It is widely acknowledged that the high-energy behaviour of quantum field theories should be governed by an ultraviolet fixed point. The fascinating idea that such a fixed point could be interacting, known as asymptotic safety, suggests that metric quantum gravity may well be fundamental in its own right

[1] D. F. Litim, Renormalisation group and the Planck scale, Phil.Trans Roy.Soc. Lond. A 369 (2011) 2759 [arXiv:1102.4624 [hep-th]].

[2] D. F. Litim and F. Sannino, Asymptotic safety guaranteed, arXiv:1406.2337 [hep-th].

[3] D. F. Litim, Fixed points of quantum gravity, Phys. Rev.Lett. 92 (2004) 201301 [hepth/0312114].

[4] K. Falls, D. F. Litim, K. Nikolakopoulos and C. Rahmede, A bootstrap towards asymptotic safet/, arXiv:1301.4191 [hep-th].

[5] K. Falls, D. F. Litim and A. Raghuraman, Black Holes and Asymptotically Safe Gravity, Int. J.Mod. Phys. A 27 (2012) 1250019 [arXiv:1002.0260 [hep-th]]. [6] K. Falls and D. F. Litim, Black hole thermodynamics under the microscope, Phys. Rev. D 89 (2014) 084002 [arXiv:1212.1821 [gr-qc]].

[7] D. F. Litim and K. Nikolakopoulos, Quantum gravity effects in Myers-Perry space-times, JHEP 1404 (2014) 021 [arXiv:1308.5630 [hep-th]].

Frontiers of Fundamental Physics 14 - FFP14,

15-18 July 2014

Aix Marseille University (AMU) Saint-Charles Campus, Marseille

\footnotetext{
*Speaker.
} 